\title{
DIRICHLET SUMMANTIONS AND PRODUCTS OVER PRIMES
}

GeOFFREY B. CAMPBELL

21 Wurruk Avenue

Preston, 3072

Victoria, Australia

(Received June 3, 1991 and in revised form July 1, 1991)

ABSTRACT. We derive new classes of infinite products taken over the primes, for example expressing

$$
\prod_{p}\left(\frac{1}{1-p^{-n}}\right)^{\left(1-p^{-m}\right)^{-1}}
$$

as an infinite produce of Riemann zeta functions, this product being taken over the set of rational numbers $\alpha / \beta$ greater than zero with $\alpha$ relatively prime to $\beta$

$$
\zeta(n) \prod_{\alpha, \beta} \zeta(\alpha m+\beta n)^{1 / \beta} .
$$

KEY WORDS AND PHRASES. Arithmetic functions, Dirichlet series and other series expansions, Convergence and divergence of infinite products, Combinatorial number theory, Lattice points in specified regions, Riemann's zeta function, Partitions (elementary number theory), Applications of sieve methods, Analytic work on partitions, Multiple sequences and series, Exponential sums, Multiplicative equations (Diophantine equations), Combinatorial identities, and Elliptic theta functions.

1991 AMS SUBJECT CLASSIFICATION CODES. 11A25, 30B10, 40A20, 11B75, 11P21, 11M06, 11P81, 11N36, 11P82, 40B05, 11L03, 11D99, 05A19, 33A25.

\section{INTRODUCTION.}

In a recent paper (Campbell [4]) a class of infinite products was derived.

The simplest of these is

$$
(1-y)_{k} \Pi_{j}\left(1-x^{j} y^{k}\right)^{1 / k}=\left(\frac{1-y}{1-x y}\right)^{1 /(1-x)}
$$

$x \neq 1,|y|<1,|x y|<1$. The notations $\prod_{j}=\prod_{k=2}^{\infty} \prod_{\epsilon \phi_{k}}{ }_{k} \sum_{j}=\sum_{k=2}^{\infty} \sum_{j \in \phi_{k}}$, where $\phi_{k}$ is the set of positive integers less than and relatively prime to $k$, are used henceforth and in (1.1). The theorem given in [4] is the case $n \rightarrow \infty$ of

THEOREM 1. If $\left(a_{k}\right)$ and $\left(b_{k}\right)$ are arbitrary sequences chosen so that, together with choice of $x$, the following functions are all defined then

$$
\sum_{k=1}^{n} a_{k} \frac{1-\exp \left(b_{k} x\right)}{1-\exp \left(b_{k} x / k\right)}=\left(\sum_{k=1}^{n} a_{k}\right)+\sum_{m=2}^{n} \sum_{k=1}^{[n / m]} \sum_{j \in \phi_{m}} a_{m k} \exp \left(b_{m k} j x / m\right),
$$

where $[n]$ denotes the greatest integer in $n$. 
The proof follows easily by induction on $n$, and resembles the proof for the infinite case given in [4]. There is an interesting parallel between this theorem and the identity used by Ramanujan ([13] and [10]) to derive his summations which generate his arithmetic function $c_{s}(n)=\sum \cos (2 \pi \lambda n / s)$ where $\lambda \in \phi_{s}$. The rigorous proof of (1.1), and also of the infinite product identities in [4], accounting for the branches of the fractional powers, can be approached easily from the finite (1.2) as $n \rightarrow \infty$.

\section{PRIME PRODUCTS FOR INFINITE PRODUCTS OF ARITHMETIC FUNCTIONS.}

There are many well known examples of functions definable in terms of an infinite or finite product of rational functions of prime number powers. Such a function is, for instance, the Riemann zeta function given by

$$
\prod_{p}\left(1-p^{-s}\right)^{-1}=\zeta(s), \quad \text { Re } s>1 .
$$

Products such as (2.1) lend themselves neatly to substitution into the new class of infinite products in [4]. To illustrate this let us take (1.1) with $x=p^{-m}, y=p^{-n}, \operatorname{Re} n>1, R e m+n>1$, and then form the product over all primes $p$ on both sides. Hence application of (2.1) yields

THEOREM 2.1. If $R e n$ and $R e m+n>1$ with $m \neq 2 r i \pi(\log p)^{-1}$ for $r \in Z$

$$
\zeta(n)_{k} \prod_{j} \zeta(j m+k n)^{1 / k}=\prod_{p}\left(\frac{1-p^{-(m+n)}}{1-p^{-n}}\right)^{\left(1-p^{-m}\right)^{-1}} .
$$

This is substantially the generalized form of

$$
\sum_{k=1}^{\infty} \psi(k) k^{-1} \log \zeta(k n)=\sum_{p}\left(p^{n}-1\right)^{-1}, \text { Re } n>1,
$$

which is given in Titchmarsh [15], where $\psi(k)$ denotes the Euler totient function. The method of deriving (2.2) is applicable to the generalized form of (1.1) given in [4] as

$$
{ }_{k} \prod_{j}\left(1-x^{j} y^{k}\right)^{j^{m} / k^{m+1}}=\exp \left\{-\zeta(m+1, y) g_{m}(x)+\sum_{k=0}^{m}\left(\begin{array}{c}
m \\
k
\end{array}\right) \zeta(1+k, x y) g_{k}(x)\right\},
$$

for $x$ and $y$ as in (1.1), $m$ any positive integer, and

$$
g_{m}(x)=\left.\frac{d^{m}}{d z^{m}}\left(1-e^{z}\right)^{-1}\right|_{z=\log x}, \quad \zeta(n, y)=\sum_{k=1}^{\infty} y^{k} k^{-n},|y|<1,
$$

for any complex number $n$. Hence we have

THEOREM 2.2. If $r \in \mathbf{Z}^{+}, R e n$ and $R e m+n>1, m \neq 2 \sin (\log p)^{-1}$ for $s \in \mathbf{Z}$ then

$$
\prod_{k} \zeta(j m+k n)^{j^{r} / k^{r+1}}=\exp \left(\sum_{p}\left\{-\zeta\left(r+1, p^{-n}\right) g_{r}\left(p^{-m}\right)+\sum_{k=0}^{r}\left(\begin{array}{l}
r \\
k
\end{array}\right) \zeta\left(1+k, p^{-(m+n)}\right) g_{k}\left(p^{-m}\right)\right\}\right)
$$

The manner of applying the Riemann zeta function to (1.1) and (2.3) works also for other functions definable by prime products. For example, the sum of the $\lambda$ th powers of the divisors of $r$, defined by

$$
\sigma_{\lambda}(r)=\prod_{i=1}^{s} \frac{p_{i}^{\left(\alpha_{i}+1\right) \lambda}-1}{p_{i}^{\lambda}-1}, \text { where } r=\prod_{i=1}^{s} p_{i}^{\alpha_{i}}
$$

can be substituted with $\lambda=-(j m+k n)$ into (1.1) and (2.3) to obtain 
THEOREM 2.3. If Re $n$ and $R e m+n>1$ with $r$ and $\sigma$ as in (2.5) then

$$
\begin{aligned}
\sigma_{-n}(r){ }_{k} \prod_{j}\left\{\sigma_{-(j m+k n)}(r)\right\}^{1 / k} & =\frac{\sigma_{n}(r)}{r^{n}} k \prod_{j}\left(\frac{\sigma_{(j m+k n)}(r)}{r^{(j m+k n)}}\right)^{1 / k} \\
& =\prod_{i=1}^{s} \rho\left(\alpha_{i}+1\right) / \rho(1), \text { with } \rho(x)=\left(\frac{1-p_{i}^{-n x}}{1-p_{i}^{-(m+n) x}}\right)^{\left(1-p_{i}^{-m x}\right)^{-1},}
\end{aligned}
$$

and if $h$ is any positive integer then

$$
\begin{gathered}
\prod_{k}\left\{\sigma_{i(j m+k n)}(r)\right\}^{j^{h} / k^{h+1}}={ }_{k} \prod_{j}\left(\frac{\sigma_{(j m+k n)}(r)}{r(j m+k n)}\right) j^{h / k^{h+1}} \\
=\prod_{i=1}^{s} \omega\left(\alpha_{i}+1\right) / \omega(1), \text { with } \\
\omega(x)=\exp \left\{-\zeta\left(h+1, p_{i}^{-n x}\right) g_{h}\left(p_{i}^{-m x}\right)+\sum_{k=0}^{h}\left(\begin{array}{l}
h \\
k
\end{array}\right) \zeta\left(k+1, p_{i}^{-(m+n) x}\right) g_{k}\left(p_{i}^{-m x}\right)\right\},
\end{gathered}
$$

$g_{h}$ and $\zeta$ defined as in (2.4). (Assuming $p_{i}^{n x} \neq 1$ ).

For example, take $m=n$ and $r=6$ and then $h=1$ so that

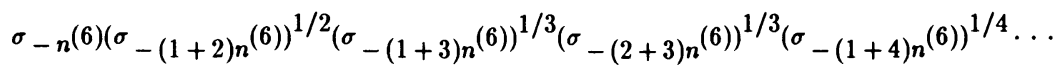

$$
\begin{aligned}
& =\left(\frac{2^{n}+1}{2^{n}}\right)^{\frac{2^{n}}{2^{n}-1}}\left(\frac{2^{2 n}}{2^{2 n}+1}\right)^{\frac{2^{2 n}}{2^{2 n}-1}}\left(\frac{3^{n}+1}{3^{n}}\right)^{\frac{3^{n}}{3^{n}-1}}\left(\frac{3^{2 n}}{3^{2 n}-1}\right)^{\frac{3^{2 n}}{3^{2 n}-1}}, \\
& \left(\sigma_{-(1+2) n}(6)\right)^{1 / 2^{2}}\left(\sigma_{-(1+3) n}(6)\right)^{1 / 3^{2}}\left(\sigma_{-(2+3) n}(6)\right)^{2 / 3^{2}}\left(\sigma_{-(1+4) n}(6)\right)^{1 / 4^{2}} \ldots \\
& =\left(\frac{2^{2 n}-1}{2^{2 n}}\right)^{\frac{2^{n}}{2^{n}-1}}\left(\frac{2^{4 n}}{2^{4 n}-1}\right)^{\frac{2^{2 n}}{2^{2 n}-1}}\left(\frac{3^{2 n}-1}{3^{2 n}}\right)^{\frac{3^{n}}{3^{n}-1}}\left(\frac{3^{4 n}}{3^{4 n}-1}\right)^{\frac{3^{2 n}}{3^{2 n}-1}} e^{A},
\end{aligned}
$$

with $A=\xi\left(2^{2 n}\right)-\xi\left(2^{n}\right)+\xi\left(3^{2 n}\right)-\xi\left(3^{n}\right)$, such that $\xi(x)$ is defined by

$$
\left(\zeta\left(2, x^{-2}\right)-\zeta\left(2, x^{-1}\right)\right) x(x-1)^{-2} .
$$

Let us next consider the Jordan's totient function $J_{k}(r)$. This is the number of ordered sets of $k$ elements chosen from a complete residue system (mod $r$ ) such that the greatest common divisor of each set is prime to $r$. It is known ([14], p. 92) that

$$
J_{k}(r)=r^{k} \prod_{p \mid r}\left(1-p^{-k}\right)
$$

Hence, again applying (1.1) and (2.3) we have

THEOREM 2.4. If $m, n$, and $r$ are as in Theorem 2.2 then

$$
\begin{gathered}
\frac{J_{n}(r)}{r^{n}} k \prod_{j}\left(\frac{J_{(j m+k n)}^{(r)}}{r^{(j m+k n)}}\right)^{1 / k}=\prod_{p \mid r}\left(\frac{1-p^{-n}}{1-p^{-(m+n)}}\right)\left(1-p^{-m}\right)^{-1}, \text { and } \\
\prod_{j}\left(\frac{J(j m+k n)^{(r)}}{r^{(j m+k n)}}\right)^{j^{h} / k^{h+1}}, \text { is for any positive integer } h, \text { equal to } \\
\exp \left(\sum_{p \mid r}\left\{-\zeta\left(h+1, p^{-n}\right) g_{n}\left(p^{-n}\right)+\sum_{k=0}^{m}\left(\begin{array}{c}
m \\
k
\end{array}\right) \zeta\left(1+k, p^{-(m+n)}\right) g_{k}\left(p^{-n}\right)\right\}\right)
\end{gathered}
$$


The main interest in the theorems of this section appears twofold. Firstly, they are examples of a new kind of infinite product taken over the primes for which an additive property for the primes is implied. That is, products of form $\prod_{p} f(p)^{g(p)}$ suggest for the first time an additive quality for the $g(p)$ function. Secondly, the operators ${ }_{k} \Pi$, and ${ }_{k} \Sigma$, define mappings from the set of rational numbers in $(0,1) 1-1$ and onto functions where, to some extent, independent variables are associated with numerator and denominator for each fraction when it is reduced into its Farey fraction form. Evidently then, Theorem 2.1 does for the set of Farey fractions in $(0,1)$ what the classical identity (2.1) does for the positive integers. Combinatorially, Euler's product (2.1) is equivalent to the statement that every positive integer is factorable into a unique product of primes. We might suspect that (2.2) implies an analogous statement for the set of Farey fractions in $(0,1)$. What hampers our making such a statement is the occurrence of fractional powers on the left side of (2.1). This issue of powers is equally serious for the right side since $\left(1-p^{-m}\right)^{-1}$ will be generally not a positive integer. However, we may start the process of understanding the left side of $(2.1)$ by considering the function $f_{j, k}(\lambda)$ where

$$
\zeta(j m+k n)^{1 / k}=\left(\sum_{\lambda=1}^{\infty} \frac{1}{\lambda^{j m+k n}}\right)^{1 / k}=\sum_{\lambda=1}^{\infty} \frac{f_{j, k}(\lambda)}{\lambda^{j m+k n}},
$$

for 'positive integers $k$ and $j \in \phi_{k}$. Clearly, (2.6) is equivalent to

$$
\sum_{\lambda_{1}=1}^{\infty} \sum_{\lambda_{2}=1}^{\infty} \sum_{\lambda_{3}=1}^{\infty} \cdots \sum_{\lambda_{k}=1}^{\infty} \frac{f_{j, k}\left(\lambda_{1}\right) f_{j, k}\left(\lambda_{2}\right) \cdots f_{j, k}\left(\lambda_{k}\right)}{\left(\lambda_{1} \lambda_{2} \lambda_{3} \cdots \lambda_{k}\right)^{j m+k n}}=\sum_{\lambda=1}^{\infty} \frac{1}{\lambda^{j m+k n}} .
$$

Since $j$ and $k$ are relatively prime in the case of (2.1) we may, in (2.7), deduce that $\lambda=\prod_{i=1}^{k} \lambda_{i}$ and that all such cases of this are included with the occurence of same factors in different orders counted as distinct. Furthermore, from (2.7), $\prod_{i=1}^{k} f_{j, k}\left(\lambda_{i}\right)$ is the reciprocal of the number of ways $\lambda$ can be split into $k$ factors in positive integers such that same factors rearranged are counted as distinct. It is evident from (2.7) that $f_{j, k}(\lambda)$ is most easily evaluable when $\lambda$ has fewest divisors. For example, it is easily deduced that for any prime $p$ we have $f_{j, k}(p)=1 / k$.

A more fundamental prime product is given by

THEOREM 2.5. If $R e n$ and $R e m>1$ then

$$
\prod_{p}\left(\frac{1}{1-p^{-n}}\right)^{\left(1-p^{-m}\right)^{-1}}=\zeta(n) \zeta(m+n){ }_{k} \prod_{j} \zeta(j m+k n)^{1 / k} \zeta(j n+k m)^{1 / j}
$$

Our proof of this involves the following definition and lemma.

DEFINITION 2.6. Let $\sigma^{+}$denote the set of all extended Farey fractions $\alpha / \beta$ such that $\alpha$ and $\beta$ are relatively prime positive integers. Furthermore, let $\sigma_{(a, b]}$ be the subset of $\sigma^{+}$in the rational interval $(a, b]$. Let this convention be adopted for any open or closed such interval.

LEMMA 2.7.

$\mathcal{F}^{+} \equiv \cup_{i=1}^{\infty} \mathscr{F ( i - 1 , i )}$

$\mathcal{F}^{+} \equiv \mathcal{F}(0,1) \cup \mathcal{F}(1, \infty) \cup\{1\}$,

$\mathscr{F}(1, \infty) \equiv\left\{\alpha / \beta \in \mathscr{F}^{+}: \beta / \alpha \in \mathscr{F}(0,1)\right\}$.

Whilst not rigorously proving this lemma, we note that its truth is linked to appropriate operations on $\mathscr{F}(0,1)$. Specifically, for 
$1 / 2$

$1 / 3 \quad 2 / 3$

$1 / 4 \quad 3 / 4$

$\begin{array}{llll}1 / 5 & 2 / 5 & 3 / 5 & 4 / 5\end{array}$

$\begin{array}{lllll}1 / 6 & 5 / 6\end{array}$

$\begin{array}{llllll}1 / 7 & 2 / 7 & 3 / 7 & 4 / 7 & 5 / 7 & 6 / 7\end{array}$

$\begin{array}{llll}1 / 8 & 3 / 8 & 5 / 8 & 7 / 8\end{array}$

etc.,

in this case of (2.9), we add a positive integer $i$ to each term in (2.12) to translate the sequence $\mathscr{F}(0,1)$ onto $\mathscr{F}(i, i+1)$, and then iterate. For $(2.10)$ and $(2.11)$ we note that the set of reciprocals of (2.12) terms is precisely the set of elements of $\mathscr{F}(1, \infty)$.

PROOF OF THEOREM 2.5. The factors on the left side of (2.2) which are subject to the operator ${ }_{k} \Pi$, may be thought of as elements listed thus

$\zeta(m+2 n)^{1 / 2}$

$\zeta(m+3 n)^{1 / 3} \quad \zeta(2 m+3 n)^{1 / 3}$

$\zeta(m+4 n)^{1 / 4}$ $\zeta(3 m+4 n)^{1 / 4}$

$\zeta(m+5 n)^{1 / 5}$

$\zeta(2 m+5 n)^{1 / 5}$

$\zeta(3 m+5 n)^{1 / 5}$

$\zeta(4 m+5 n)^{1 / 5}$

$\zeta(m+6 n)^{1 / 6}$

$\zeta(m+7 n)^{1 / 7}$

$\zeta(2 m+7 n)^{1 / 7}$

$\zeta(3 m+7 n)^{1 / 7}$

$\zeta(4 m+7 n)^{1 / 7}$

$\zeta(5 m+6 n)^{1 / 6}$

etc.,

of a set which is the result of an isomorphic mapping from $\mathscr{F}(0,1)$. This is immediately seen by comparison with (2.12). Let $P(m, n)$ be the left side of $(2.1)$ so that the term factors of $P(m, n+r m)$ for $r$ any positive integer will correspond exactly to the elements of $\Im(r, r+1]$. Hence, applying (2.2) and $(2.9)$ to the product $\prod_{i=1}^{r-1} P(m, m i+n)$ we have

$$
\prod_{p}\left(\frac{1-p^{-(r m+n)}}{1-p^{-n}}\right)^{\left(1-p^{-m}\right)^{-1}}=\zeta(n) \zeta(m+n) \Pi^{\prime} \zeta(\alpha m+\beta n)^{1 / \beta},
$$

where $\Pi^{\prime}$ is over $\alpha, \beta$ respective numerators and denominators of the fractions in $\Im(0, r]$. If we let $r \rightarrow \infty$ in (2.14), the right side product is then over all $\alpha / \beta$ being elements of $\sigma^{+}$. It remains for us to establish that this product taken over $\mathcal{F}^{+}$is equivalent to the right side product in (2.8). (2.10) and (2.11) show exactly this as required and so Theorem 2.5 is proved.

We note that if $m=n$ in Theorem 2.5 we have

COROLLARY 2.8. If $R e m>1$ then

$$
\prod_{p}\left(\frac{1}{1-p^{-m}}\right)^{\left(1-p^{-m}\right)^{-1}}=\prod_{k=1}^{\infty} \zeta(k m)^{k \Sigma^{\prime}(\alpha \beta)^{-1}}
$$

where $\Sigma^{\prime}$ is over all positive integers $\alpha, \beta$ which are relatively prime and whose sum is $k$.

It is worth noting that (2.16) and (2.17) are equivalent expressions for the left side of (2.8): -

$$
\exp \left\{\sum \frac{1}{\mu p^{\lambda m+\mu n}}\right\}
$$


where the summation is over all primes $p$, and all positive integers $\lambda+1, \mu$.

$$
\prod_{\lambda, \mu} \sum_{r=1}^{\infty} \frac{1}{\left(\prod_{i=1}^{s} \alpha_{i} !\right) \mu^{s_{r}} \lambda m+\mu n}, \text { where } r=\prod_{i=1}^{s} p_{i}^{\alpha_{i}}
$$

which is the prime product decomposition of $r$, and with the left-most product over all positive integers $\lambda+1, \mu$.

\section{DIRICHLET SUMMATIONS AND A GENERALIZED TOTIENT FUNCTION.}

In this section we examine the identity which arises from equating coefficients of like powers of $x$ in the expansion of Theorem 1 . Without loss of generality we let $b_{k}=1$ for all positive integers $k$, and use the following

DEFINITION 3.1. For all positive integers $k-1$ and $m$, let

$$
\psi_{m}(k)=\sum_{\lambda \in \phi_{k}}(\lambda / k)^{m}
$$

We note that if $m$ were zero in (3.1) it would define the Euler totient function for $k \geq 2$. Theorem 1 expanded as power series in $x$ leads to

THEOREM 3.2 .

$$
\begin{aligned}
\psi_{m}(2) \sum_{1}^{[n / 2]} a_{2 k}+\psi_{m}(3) \sum_{1}^{[n / 3]} a_{3 k}+\psi_{m}(4) \sum_{1}^{[n / 4]} a_{4 k}+\cdots \\
=\sum_{\lambda=2}^{n}\left(\psi_{m}(\lambda) \sum_{k=1}^{[n / \lambda]} a_{\lambda k}\right) \\
=\sum_{k=1}^{n}\left\{k a_{k} \sum_{\lambda=0}^{m} \frac{B_{\lambda}}{(m+1-\lambda) k^{\lambda}}\left(\begin{array}{c}
m \\
\lambda
\end{array}\right)\right\}
\end{aligned}
$$

where $B_{\lambda}$ are the Bernoulli numbers and $\left(a_{k}\right)$ is any sequence for which all the functions are defined.

This theorem leads simply to many corollaries; often analogous to the identities in Ramanujan [13]. Of particular interest is the case $n \rightarrow \infty, a_{k}=k^{-s}$, yielding

COROLLARY 3.3. (Lossers [12])

$$
\sum_{k=2}^{\infty} \psi_{m}(k) k^{-s}=\zeta(s)^{-1} \sum_{\lambda=0}^{m}\left(\begin{array}{c}
m \\
\lambda
\end{array}\right) \frac{B_{\lambda} \zeta(s+\lambda-1)}{m+1-\lambda}, \quad \text { Re } s^{\prime}>2 .
$$

If, in this, we note that

$$
\zeta(s)^{-1} \zeta(s+\lambda-1)=\prod_{p} \frac{1-p^{-s}}{1-p^{1-s-\lambda}}=1+\sum_{k=1}^{\infty} k^{1-\lambda} \prod_{p \mid k}\left(1-p^{\lambda-1}\right) k^{-s},
$$

we get the closed form of $\psi_{m}(k)$ in terms of the prime divisors of $k$,

COROLLARY 3.4.

$$
\psi_{m}(k)=\sum_{\lambda=0}^{m}\left(\begin{array}{c}
m \\
\lambda
\end{array}\right) \frac{B_{\lambda} k^{1-\lambda}}{m+1-\lambda} \prod_{p \mid k}\left(1-p^{\lambda-1}\right)=\sum_{\lambda=0}^{m}\left(\begin{array}{c}
m \\
\lambda
\end{array}\right) B_{\lambda} J_{1-\lambda}(k)(1-\lambda+m)^{-1} .
$$

From Definition 3.1 we see that

$$
\sum_{d \mid k} \psi_{m}(d)=\sum_{\lambda=1}^{k}(\lambda / k)^{m}
$$


Some simple cases of Theorem 3.2 are given by

COROLLARY 3.5.

$$
\begin{aligned}
& \psi_{m}(2)[n / 2]+ \psi_{m}(3)[n / 3]+\psi_{m}(4)[n / 4]+\cdots \\
&=\left(\begin{array}{c}
m \\
0
\end{array}\right) \frac{B_{0}}{l+m} \frac{n(n+1)}{2}+\left(\begin{array}{c}
m \\
1
\end{array}\right) \frac{B_{1}}{m} n+\left(\begin{array}{c}
m \\
2
\end{array}\right) \frac{B_{2}}{m-1} \sum_{1}^{n} k^{-1}+\left(\begin{array}{c}
m \\
3
\end{array}\right) \frac{B_{3}}{m-2} \sum_{1}^{n} k-2+\cdots, \\
& \psi_{m}(2)[n / 2]^{2}+\psi_{m}(3)[n / 3]^{2}+\psi_{m}(4)[n / 4]^{2}+\cdots \\
&=2 \\
&\left.\quad\left\{\begin{array}{c}
m \\
0
\end{array}\right) \frac{B_{0}}{1+m} \frac{n(n+1)(2 n+1)}{6}+\left(\begin{array}{c}
m \\
1
\end{array}\right) \frac{B_{1}}{m} \frac{n(n+1)}{2}+\left(\begin{array}{c}
m \\
2
\end{array}\right) \frac{B_{2}}{m-1} n+\left(\begin{array}{c}
m \\
3
\end{array}\right) \frac{B_{3}}{m-2} \sum_{1}^{n} k^{-1}+\cdots\right\} \\
& \quad-\left\{\left(\begin{array}{c}
m \\
0
\end{array}\right) \frac{B_{0}}{1+m} \frac{n(n+1)}{2}+\left(\begin{array}{c}
m \\
1
\end{array}\right) \frac{B_{1}}{m} n+\left(\begin{array}{c}
m \\
2
\end{array}\right) \frac{B_{2}}{m-1} \sum_{1}^{n} k^{-1}+\left(\begin{array}{c}
m \\
3
\end{array}\right) \frac{B_{3}}{m-2} \sum_{1}^{n} k-2+\cdots\right\}
\end{aligned}
$$

$$
\begin{aligned}
& \psi_{m}(2) \sum_{1}^{[n / 2]}(2 k)^{s}+\psi_{m}(3) \sum_{1}^{[n / 3]}(3 k)^{s}+\psi_{m}(4) \sum_{1}^{[n / 4]}(4 k)^{s}+\cdots \\
& =\left(\begin{array}{c}
m \\
0
\end{array}\right) \frac{B_{0}}{1+m} \sum_{1}^{n} k^{s+1}+\left(\begin{array}{c}
m \\
1
\end{array}\right) B_{1} \frac{1}{m} \sum_{1}^{n} k^{s}+\left(\begin{array}{c}
m \\
2
\end{array}\right) \frac{B_{2}}{m-1} \sum_{1}^{n} k^{s-1}+\cdots, \\
& \psi_{m}(2) x^{2} \frac{1-x^{2[n / 2]}}{1-x^{2}}+\psi_{m}(3) x^{3} \frac{1-x^{3[n / 3]}}{1-x^{3}}+\psi_{m}(4) x^{4} \frac{1-x^{4[n / 4]}}{1-x^{4}}+\cdots \\
& =\left(\begin{array}{c}
m \\
0
\end{array}\right) \frac{B_{0}}{1+m} \frac{x-(n+1) x^{n+1}+n x^{n+2}}{(1-x)^{2}}+\left(\begin{array}{c}
m \\
1
\end{array}\right) \frac{B_{1}}{m} \frac{x-x^{n+1}}{1-x}+\left(\begin{array}{c}
m \\
2
\end{array}\right) \frac{B_{2}}{m-1} \sum_{1}^{n} k^{-1} x^{k}+\cdots,
\end{aligned}
$$

for any value of $s$ and $x$ except for $|x|=1$; each series being finite to $n-1$ terms on the left sides, and to $m+1$ terms on the right sides.

A notable case of Theorem 3.2 is with $n \rightarrow \infty, a_{k}=y^{k} k^{-1}$, yielding

COROLLARY 3.6. (Campbell [4]) If $|y|<1$ and $f(k, y)=\sum_{j=1}^{\infty} j^{-k_{y} j}$

$$
\prod_{k=2}^{\infty}\left(1-y^{k}\right)^{\psi_{m}(k) / k}={ }_{k} \prod_{j}\left(1-y^{k}\right)^{j^{m} / k^{m+1}}=\exp \left\{\sum_{k=0}^{m}\left(\begin{array}{c}
m \\
k
\end{array}\right) B_{k} f(k, y)\right\}
$$

This result is unusual since the functions $\alpha(k)$ and $\beta(k)$ defined by

$$
\prod_{k=2}^{\infty}\left(1-y^{k}\right)^{\psi_{m}(k) / k}=\sum_{k=0}^{\infty} \alpha(k) y^{k}=\left\{\sum_{k=0}^{\infty} \beta(k) y^{k}\right\}^{-1},
$$

are easily computed in finite form from (3.9). Elaborate classical methods are normally applied to such problems. For example, asymptotic estimates for the generated coefficients of the function $\prod_{k=1}^{\infty}\left(1-y^{k}\right)^{-a_{k}}$ with $a_{k}$ non-negative real numbers are treated in Andrews [2], ch. 6. Thus, $\beta(k)$ can be found exactly from Corollary 3.6, whilst the theorem of Meinardus will yield an asymptotic estimate. We leave a comparative study of these results for a later paper.

Let the Definition 3.1 extend to include all complex numbers $m$. Then

THEOREM 3.7. If $R e m$ and $R e n>1$ then

$$
1+\sum_{k=2}^{\infty} \frac{\psi_{-m}(k)+\psi_{-n}(k)}{k^{m+n}}=\frac{\zeta(m) \zeta(n)}{\zeta(m+n)} .
$$


PROOF. If $R e m$ and $R e n>1$ then

$$
\begin{aligned}
& \zeta(m) \zeta(n) \\
&=\left(1+\left(1^{-m}+2^{-m}\right) 2^{-n}+\left(1^{-m}+2^{-m}+3^{-m}\right) 3^{-n}+\left(1^{-m}+2^{-m}+3^{-m}+4^{-m}\right) 4^{-n}+\cdots\right) \\
&+\left(1+\left(1^{-n}+2^{-n}\right) 2^{-m}+\left(1^{-n}+2^{-n}+3^{-n}\right) 3^{-m}+\left(1^{-n}+2^{-n}+3^{-n}+4^{-n}\right) 4^{-m}+\cdots\right) \\
&-\zeta(m+n) \\
&= \zeta(m+n)\left\{\left(1+\psi_{-m}(2) 2^{-m-n}+\psi_{-m}(3) 3^{-m-n}+\psi_{-m}(4) 4^{-m-n}+\cdots\right)\right. \\
&\left.+\left(1+\psi_{-n}(2) 2^{-m-n}+\psi_{-n}(3) 3^{-m-n}+\psi_{-n}(4) 4^{-m-n}+\cdots\right)\right\}-\zeta(m+n),
\end{aligned}
$$

so that (3.10) follows trivially. End of proof.

Next we consider the case of Theorem 1 with $n \rightarrow \infty$ and $b_{k}=x^{-1} \log q$,

COROLLARY 3.8. If $\left(a_{k}\right)$ and $q$ are suitably chosen then

$$
\sum_{k=1}^{\infty} a_{k} \frac{1-q}{1-q}=S_{1}+\sum_{m=2}^{\infty}\left(S_{m} \sum_{j \in \phi_{m}} q^{j / m}\right), \text { where } S_{m}=\sum_{k=1}^{\infty} a_{k m} \text {. }
$$

In particular, for positive integers $n$, if $q=\exp (2 \pi i n)$,

$$
\sum_{j \in \phi_{k}} q^{j / k}=\sum_{j \in \phi_{k}} \cos \left(\frac{2 \pi n j}{k}\right)=c_{k}(n)
$$

namely, Ramanujan's trigonometrical function. (see [13] primarily, also [9], [11], [14], and [15]). Hence, substituting this value for $q$ into (3.11) and applying the well known summation,

$$
\sum_{j=0}^{r-1} \exp \left(\frac{2 \pi i n j}{r}\right)=\left\{\begin{array}{l}
r \text { if } r \mid n \\
0 \text { otherwise }
\end{array}\right.
$$

to the left side of (3.11), we arrive at

COROLLARY 3.9. If $\left(a_{k}\right)$ is suitably chosen then

$$
\sum_{r \mid n} r a_{r}=\sum_{k=1}^{\infty} S_{k} c_{k}(n), \text { where } S_{m}=\sum_{j=1}^{\infty} a_{m j}
$$

Many of the summations in Ramanujan's [9] are simple cases of this, including, for $a_{k}=k^{-s-1}$, the classical result

$$
\sigma_{-s}(n) \zeta(s+1)^{-1}=\sum_{k=1}^{\infty} c_{k}(n) k^{-s-1}, \quad \operatorname{Re} s>1 .
$$

We may, of course, select $\left(a_{k}\right)$ such that $S_{m}$ of (3.12) is a finite sum with $a_{k}=0$ for $k$ greater than a certain integer $j$. In that case, $S_{m}$ will be summed over $[j / m]$ terms. Another simple case of (3.12) is

$$
\exp \left\{\sum_{r \mid n} y^{r}\right\}=\prod_{k=1}^{\infty}\left(1-y^{k}\right)^{-c_{k}(n) / k}, \quad|y|<1 .
$$

As with Corollary 3.6, the coefficients generated from the infinite product of (3.14) are easily calculated in finite form.

The results of this section work out most neatly wherever the summations $S_{m}$ together with the functions $a_{m k}$ are simply and finitely evaluable. It is well known that if $f(k)$ is a multiplicative function, that is, $f(m n)=f(m) f(n)$ whenever $(m, n)=1$, then any existing $\Sigma_{1}^{\infty} f(k) k^{-s}$ is a product of 
Riemann zeta functions. In that case, if $m$ is any positive integer then $\Sigma_{1}^{\infty} f(m k) k^{-s}$ can be evaluated. For example, $a_{k}=\sigma_{a}(k) k^{-s}$ with $R e s-a>1$, leads to

$S_{m}=\sum_{k=1}^{\infty} \sigma_{a}(m k)(m k)^{-s}=\zeta(s) \zeta(s-a) m^{-s} \prod_{i=1}^{t} \frac{1-p_{i}^{a-s}-p_{i}^{\left(\alpha_{i}+1\right) a}+p_{i}^{\left(\alpha_{i}+1\right) a-s}}{1-p_{i}^{a}}$,

where $m=\Pi_{i=1}^{t} p_{t}^{\alpha_{i}}$. This is derived from taking the product over primes $p_{i}$ for the associated function $\sum_{k=0}^{\infty} \sigma_{a}\left(p_{i}^{\alpha}+k\right) p_{i}^{-k s}$.

The transform which maps the function $a_{k}$ onto its image function $S_{m}$ appears to be most easily found when: series.

(i) $a_{k}=f(k) k^{-s}$ so that $S_{m}$ is found from the theory of prime products and their Dirichlet

(ii) $a_{k}=g(k) q^{k}$ so that evaluating $S_{m}$ depends on some application of the Hecke operator (see Andrews [2], p. 161)

$$
\text { น }_{m}\{F(q)\}=\sum_{k=-\infty}^{\infty} a_{m k} q^{k}=m^{-1} \sum_{j=0}^{m-1} F\left(\omega^{j} q^{1 / m}\right),
$$

where $\omega=\exp (2 \pi i / m)$ and $F(q)=\sum_{k=-\infty}^{\infty} a_{k} q^{k}$, this latter mermorphic around $q=0$.

Thirdly, but less generally, a combination of (i) and (ii) has led to the infinite products in Çampbell [4].

\section{LAMBERT SERIES AND ARITHMETIC FUNCTIONS.}

So far we have considered Theorem 1 for cases where $b_{k}=1$ or $b_{k}=k$. Now we examine cases with $\left(b_{k}\right)$ an increasing sequence of positive integers other than the $b_{k}=k$ already considered. We especially consider cases of Theorem 1 where $a_{k}=1$ so that convergence of the infinite series cases depend on the $\left(b_{k}\right)$ sequence. The results of greatest interest in this section come apparently from cases of Theorem 1 with $n \rightarrow \infty$. However, the convenience of the finite form of Theorem 1 is that all of the infinite versions of the theorem can be rigorously proven by elementary processes. The following theorem is simply a restatement of Theorem 1 with $n \rightarrow \infty$.

THEOREM 4.1. If $\left(a_{k}\right)$ and $\left(b_{k}\right)$ are arbitrary sequences of functions which, with the variable $q$, are chosen for convergence of the following, then

$$
\sum_{k=1}^{\infty} a_{k} \frac{1-q^{k b_{k}}}{1-q^{b} k}=\sum_{k=1}^{\infty}\left\{a_{k}+\sum_{m=2}^{\infty} \sum_{j \in \phi_{m}} a_{m k} q^{j k b_{m k}}\right\} .
$$

This theorem is the key to the results of this section. In order to use (4.1) with $a_{k}=1$ we note that it may be rewritten as

$$
\sum_{k=1}^{\infty} a_{k} \frac{q^{b_{k}-q^{k} b_{k}}}{1-q^{b_{k}}}=\sum_{k=1}^{\infty} \sum_{m=2}^{\infty} \sum_{j \in \phi_{m}} a_{m k} q^{j k b_{m k}}=\sum_{k=1}^{\infty} m \sum_{j} a_{m k} q^{j k b_{m k}},
$$

using the notation of $\$ 1$. When $b_{k}=k$ in Theorem 4.1 or the (4.2) version of it, the identities resulting often involve Jacobi theta functions. Many such related identities are relevant also to the theory of partitions. For example,

THEOREM 4.2. For $z \neq 0$ and $|q|<1,|z q|<1$ with $|z| \geq 1$,

where

$$
\sum_{k=1}^{\infty}\left(z^{k}+z^{-k}\right) \frac{q^{k}-q^{k^{2}}}{1-q^{k}}={ }_{\lambda} \sum_{\mu}\left\{\Pi\left(z^{\lambda}, q^{\lambda \mu}\right)-1\right\}
$$

$$
\sum_{k=-\infty}^{\infty} z^{k} q^{k^{2}}=\prod_{k=0}^{\infty}\left(1-q^{2 k+2}\right)\left(1+z q^{2 k+1}\right)\left(1+z^{-1} q^{2 k+1}\right):=\Pi(z, q)
$$


PROOF. Let $a_{k}=z^{k}+z^{-k}$ and $b_{k}=k$ in the (4.2) form of Theorem 4.1. The left side of (4.3) compares for convergence with $\Sigma_{1}^{\infty}\left(z^{k}+z^{-k}\right) q^{k}$ which converges only if $\lim _{k \rightarrow \infty}\left(z^{k}+z^{-k}\right) q^{k}=0$. Clearly then $z$ and $q$ must fulfill the conditions $\left|z^{k} q^{k}\right| \rightarrow 0$ as $k \rightarrow \infty$ assuming $|z| \geq 1$ with $|q|<1$. End of proof.

Theorem 4.3 resembles Theorem 4.2. We follow this with a definition and some simple theta function identities.

THEOREM 4.3. For $z \neq 0$ and $|q|<1$, Re $a>0$,

$$
\begin{aligned}
& \sum_{k=1}^{\infty}\left(z^{k}+z^{-k}\right) q^{a k^{2}} \frac{1-q^{k^{2}}}{1-q^{k}}=\prod\left(z, q^{a}\right)-1+{ }_{\lambda} \sum_{\mu}\left(\prod\left(z^{\lambda}, q^{\lambda(\lambda a+\mu)}\right)-1\right),
\end{aligned}
$$

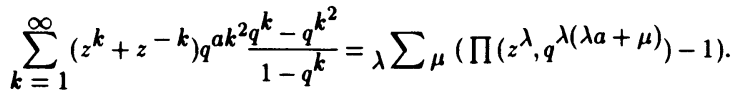

DEFINITION 4.4. For $|q|<1$, let

$$
\begin{aligned}
& \Theta_{1}(z, q)=\sum_{k=1}^{\infty} q^{k^{2}} \cos 2 k z, \quad \Theta_{2}(z, q)=\sum_{k=1}^{\infty} q^{k^{2}} \sin 2 k z \\
& \Theta_{1}(z, q)=\Theta_{1}(z)=2 q^{\frac{1}{4}} \sum_{k=0}^{\infty}(-1)^{k} q^{k(k+1)} \sin (2 k+1) z .
\end{aligned}
$$

THEOREM 4.5. If $|q|<1$, and in $(4.5) z \neq\left(n+\frac{1}{2}\right) \pi$ for integers $n$,

$$
\begin{aligned}
& \sum_{k=1}^{\infty} \frac{q^{2 k^{2}}}{1-q^{2 k}} \sin 2 k z=\frac{1}{4}\left(\frac{\Theta_{1}^{\prime}(z)}{\Theta_{1}(z)}-\cot z\right)+{ }_{k} \sum_{j} \Theta_{2}\left(k z, q^{2 j k}\right), \\
& \sum_{k=1}^{\infty} \frac{q^{k^{2}}}{1-q^{k}} \cos 2 k z=\left(\sum_{k=1}^{\infty} \frac{q^{k}}{1-q^{k}} \cos 2 k z\right)-{ }_{k} \sum_{j} \Theta_{1}\left(k z, q^{j k}\right) .
\end{aligned}
$$

PROOF. To obtain (4.5), (4.6), respectively substitute in Theorem 4.1 the values $a_{k}=\sin 2 k z$, $\cos 2 k z$, and $b_{k}=2 k, k$, then use the fact that

$$
\frac{\Theta_{1}^{\prime}(z)}{\Theta_{1}(z)}=\cot z+4 \sum_{k=1}^{\infty} \frac{q^{2 k}}{1-q^{2 k}} \sin 2 k z .
$$

Use of the notation in Definition 4.4 concludes the proof.

The identity (see [1], 16.30.1)

$$
\sum_{k=1}^{\infty} \frac{1}{k} \frac{q^{2 k}}{1-q^{2 k}} \sin 2 k \alpha \sin 2 k \beta=\frac{1}{4} \log \frac{\Theta_{1}(\alpha+\beta) \sin (\alpha-\beta)}{\Theta_{1}(\alpha-\beta) \sin (\alpha+\beta)},
$$

lends itself neatly to Corollary 3.8 rather than to (4.1) or (4.2). In that case it seems worth noting that if $|q|<1, x^{1 / k} \neq 1$, then

$$
\begin{aligned}
\exp \left(4 \sum_{k=1}^{\infty} \frac{1}{k} \frac{q^{2 k}}{1-q^{2 k}}\right. & \left.\frac{1-x}{1-x^{1 / k}} \sin 2 k \alpha \sin 2 k \beta\right) \\
& =\frac{\Theta_{1}(\alpha+\beta) \sin (\alpha-\beta)}{\Theta_{1}(\alpha-\beta) \sin (\alpha+\beta)} \prod_{k=1}^{\infty}\left(\frac{\Theta_{1}\left((\alpha+\beta) k, q^{2 k}\right) \sin (\alpha-\beta) k}{\Theta_{1}\left((\alpha-\beta) k, q^{2 k}\right) \sin (\alpha+\beta) k}\right)^{f_{k}(x) / k},
\end{aligned}
$$

where $f_{k}(x)=\sum_{\lambda \in \phi_{k}} x^{\lambda / k}$. In particular for positive integers $n$ with $x=\exp (2 \pi i n)$ we have

$\exp \left(4 \sum_{k \mid n} \frac{q^{2 k}}{1-q^{2 k}} \sin 2 k \alpha \sin 2 k \beta\right)$ 


$$
\begin{aligned}
& \text { DIRICHLET SUMMATIONS AND PRODUCTS OVER PRIMES } \\
& =\frac{\Theta_{1}(\alpha+\beta) \sin (\alpha-\beta)}{\Theta_{1}(\alpha-\beta) \sin (\alpha+\beta)} \prod_{k=1}^{\infty}\left(\frac{\Theta_{1}\left((\alpha+\beta) k, q^{2 k}\right) \sin (\alpha-\beta)}{\Theta_{1}\left((\alpha-\beta) k, q^{2 k}\right) \sin (\alpha+\beta)}\right)^{c_{k}(n) / k},
\end{aligned}
$$

where $c_{k}(n)$ is Ramanujan's trigonometric function as in $\S 3$.

The following generalization of Theorems 4.2 and 4.3 seems of combinatorial interest.

THEOREM 4.6. If $|q|<1, a \geq 0, m$ any positive integer, $z$ any complex number except for the case $a=0, m=1$, which is essentially Theorem 4.2 ,

$$
\sum_{k=1}^{\infty} z^{k} q^{a k^{m+1}} \frac{q^{k^{m}}-q^{k^{m+1}}}{1-q^{k^{m}}}={ }_{\lambda} \sum_{\mu} \Xi_{m}\left(z^{\lambda}, q^{\lambda^{m}(a \lambda+\mu)}\right)
$$

where $\Xi_{m}(z, q)=\sum_{k=1}^{\infty} z^{k} q^{k^{m+1}}$

PROOF. We require (4.2) with substitutions $a_{k}=z^{k} \exp \left(a k^{m+1} \log q\right), b_{k}=k^{m}$ with the conditions specified for the theorem. Note that convergence for cases with $m$ integers greater than 1 , the $q$ powers dominate. For $m=1,(4.9)$ is closely related to Theorem 4.3 , and its proof is only trivially different in content. However, for $m=2,3,4, \ldots$ we see that

$$
\begin{aligned}
& \sum_{k=1}^{\infty} z^{k} q^{a k^{m+1}} \frac{q^{k^{m}}-q^{k^{m+1}}}{1-q^{k^{m}}} \\
& =\sum_{k=1}^{\infty}\left\{z^{2 k} q^{a(2 k)^{m+1}} q^{1.2^{m} k^{m+1}}+z^{3 k} q^{a(3 k)^{m+1}}\left(q^{1.3^{m} k^{m+1}}+q^{2.3^{m} k^{m+1}}\right)\right. \\
& \left.+z^{4 k} q^{a(4 k)^{m+1}}\left(q^{1.4^{m} k^{m+1}}+q^{3.4^{m} k^{m+1}}\right)+\cdots\right\} \\
& ={ }_{\lambda} \sum_{\mu} \sum_{k=1}^{\infty} z^{\lambda k} q^{\lambda^{m}(a \lambda+\mu) k^{m+1}} \\
& ={ }_{\lambda} \sum_{\mu} \Xi_{m}\left(z, q^{\lambda^{m}(a \lambda+\mu)}\right) \text {. End of proof. }
\end{aligned}
$$

We now consider some combinatorial inferences from the theorems in this section. It may also be of interest to interpret identities (4.7) and (4.8). However, the theorems of this section yield simple relations between the generated coefficients, so for brevity we focus on these only. Examples of such relations are found in, for instance, [10] and [11].

THEOREM 4.7. Let $\left(a_{k}\right)$ and $\left(b_{k}\right)$ be sequences of positive integers such that for $|q|<1$,

$$
\sum_{k \geq 1} q^{a_{k}+b_{k}} /\left(1-q^{b_{k}}\right)
$$

converges. Also, let $\alpha(A), \beta(B), \gamma(C)$ be respectively the number of solutions of the independent Diophantine equations, $A=a_{k}+n b_{k}, B=a_{k}+(n-1+k) b_{k}, C=a_{m k}+j k b_{m k}$, for fixed positive integers $A, B, C$, and various positive integers $m-1, n, j \in \phi_{m}$, $k$. Then for each positive integer $h$ we have $\alpha(h)=\beta(h)+\gamma(h)$.

PROOF. Select $\left(a_{k}\right) \rightarrow \exp \left(\left(a_{k}\right) \log q\right)$ in (4.2). Then we have

$$
\sum \frac{q^{a_{k}+b_{k}}}{1-q^{b_{k}}}=\sum \frac{q^{a_{k}+k b_{k}}}{1-q^{b_{k}}}+\sum q^{a_{m k}+j k b_{m k}},
$$


where the first two sums are taken over the positive integers $k$, whilst the third summation is over $m, j$, and $k$ as defined in Theorem 4.7. We also see that (4.10) must converge for us to expand (4.11) to obtain

$$
\sum q^{a_{k}+n b} k=\sum q^{a_{k}+(n-1+k) b} k+\sum q^{a_{m k}+j k b} m k
$$

which is equivalent to, for summations over positive integers $h$,

$\sum \alpha(h) q^{h}=\sum \beta(h) q^{h}+\sum \gamma(h) q^{h}$. End of proof.

Although $\left(a_{k}\right)$ and $\left(b_{k}\right)$ of Theorem 4.7 are arbitrary enough to include the prime numbers and some arithmetic functions, the theorem is best applied to sequences whose $m k$ th term is neatly specified. Such a function is $k^{r}$ with $r \geq 1$. If $a_{k}$ is any constant positive integer it cancels from the identity, and the $\left(b_{k}\right)$ sequence becomes all-important. Hence, choice of $b_{k}=k^{r}$ with $a_{k}=$ constant yields

COROLLARY 4.8. For every positive integer $h$,

$$
\alpha(h)=\beta(h)+\gamma(h),
$$

where $\alpha(h)=N\left\{h=n k^{r}\right\}, \beta(h)=N\left\{h=(n-1+k) k^{r}\right\}, \gamma(h)=N\left\{h=j k^{r+1} m^{r}\right.$ with $\left.j \in \phi_{m}\right\}$, with $N\{\cdots\}$ the number of positive integer solutions of $\cdots$, and $r$ any positive integer.

A further notable example of Theorem 4.7 with its Theorem 4.1 counterpart is the case $a_{k}=$ constant, $b_{k}=(k-1) !$, yielding

COROLLARY 4.9. If $|q|<1$ then

$$
\sum_{k=1}^{\infty} \frac{q^{(k-1) !}-q^{k !}}{1-q^{(k-1) !}}={ }_{m} \sum_{j} \sum_{k=1}^{\infty} q^{(m k) ! j / m},
$$

so that

$N\{h=n(k-1) !\}=N\{h=(n-1)(k-1) !+k !\}+N\left\{h=(m k) ! j / m\right.$ with $\left.j \in \phi_{m}\right\}$.

Clearly, there are numerous other identities examinable using the methods of this section. A further way of obtaining Lambert series from Theorem 4.1 is adopt a similar approach to that of Section 3. Since any Dirichlet summation has a Lambert series counterpart, the method outlined at the end of Section 3 for Dirichlet series $S_{m}$ will also yield Lambert series transformations. An adjunct to this may be the work of Apostol and Zuckerman [3] on the functional equation for multiplicative functions,

$$
F(m n) F((m, n))=F(m) F(n) f((m, n)) .
$$

However, generally the Lambert series is less manageable than the corresponding Dirichlet summation.

5. MORE INFINITE PRODUCTS, AND WEIGHTED VECTOR PARTITIONS.

The method of Section 2 which takes us from Theorem 2.1 to Theorem 2.5 is applicable to the more fundamental identity (1.1). An "isomorphic" line of reasoning involving iteration on (1.1) with $y$ replaced successively by $x y, x^{2} y, x^{3} y, \ldots, x^{n} y$; forming the finite product of these cases, then letting $n \rightarrow \infty$, yields

THEOREM 5.1 If $|x|$ and $|y|<1$,

$$
(1-y)(1-x y){ }_{k} \prod_{j}\left(1-x^{j} y^{k}\right)^{1 / k}\left(1-x^{k} y^{j}\right)^{1 / j}=(1-y)^{1 /(1-x)} .
$$


The left side of this theorem clearly contains all factors of the left side of (1.1), hence the companion identities

THEOREM 5.2. For $|y|<1,|x y|<1, x \neq 1$,

$$
(1-y)_{k} \prod_{j}\left(1-x^{j} y^{k}\right)^{1 / k}=\left(\frac{1-y}{1-x y}\right)^{1 /(1-x)},
$$

whilst for $|x|<1,|x y|<1$,

$$
(1-x y)_{k} \prod_{j}\left(1-x^{k} y^{j}\right)^{1 / j}=(1-x y)^{1 /(1-x)} .
$$

We note that Theorem 5.1 leads to Theorem 2.5 if we suitably substitute $x=p^{-m}, y=p^{-n}$, and form the Euler product over primes $p$ on both sides. This technique and similar variations on it will give, from (5.3), companion identities for Theorems 2.1 through to 2.4. We note also that the range for the variable $y$ in (5.3) has been extended trivially from that of $|y|<1$ to $|x y|<1$. It seems of particular interest to observe that the exponent $1 /(1-x)$ on the right sides of $(5.1)$ to (5.3) may be selected as a positive integer $n$ whenever $x=1-1 / n$. In such cases, the right sides are: very simple power series for (5.2), and polynomials in $y$ for (5.1) and (5.3). This leads to the expectation that these identities might have "near bijection" proofs as does the well known Euler's pentagonal number identity (see Andrews [2])

$$
\prod_{n=1}^{\infty}\left(1-q^{n}\right)=\sum_{m=-\infty}^{\infty}(-1)^{m} q^{\frac{1}{2} m(3 m-1)}, \quad|q|<1 .
$$

Clearly (5.1) and (5.3) will suggest companion identities to those given in Campbell [4], and each such identity will have a combinatorial interpretation in terms of two dimensional vector partitions. (see ch. 12 of Andrews [2]).

For example, the coefficient of $x^{A} y^{B}$ in the left side of (5.1) is

$$
\sum \prod_{i=1}^{s}(-1)^{m+n+\beta_{i}}\left(\begin{array}{c}
1 / \beta_{i} \\
B_{i}
\end{array}\right),
$$

where the summation is over solutions of the two dimensional vector equation

$$
m(0,1)+n(1,1)+\sum_{i=1}^{s_{j}} B_{i}\left(\alpha_{i}, \beta_{i}\right)=(A, B),
$$

where $m$ and/or $n$ can be 0 or, $B_{i}$ and $s_{j}$ are positive integers, whilst $\alpha_{i}$ and $\beta_{i}$ are positive relatively prime integers. The sum (5.5) taken over (5.6) is obviously not easily amenable to calculation, and yet the right side of (5.1) tells us it is in fact equal to

$$
\left.(-1)^{B} \text { \{coefficient of } x^{A} \text { in }\left(\begin{array}{c}
1 /(1-x) \\
B
\end{array}\right)\right\}
$$

\section{CONCLUSION}

The author has left many aspects of this paper open-ended. For example, the bivariate Dirichlet series resulting from $\$ 2$ will be developed in a future paper, as will the vector partition interpretations suggested by $\$ 5$. Although Theorem 3.2 can be derived simply (and without Theorem 1) using the inclusion-exclusion principle, the results of that section may link with the $q$ analogue idea for the Euler totient function in Campbell [4]. $\$ 4$ contains work mainly due to 
Theorem 1, and there may be scope for further development along similar lines. The companion identities in $\$ 5$ seem to suggest an entirely new type of identity to research.

ACKNOWLEDGEMENT. The author wishes to thank Professor George E. Andrews for his facilitating submission of this paper.

\section{REFERENCES}

1. ABRAMOWITZ, M.A. \& STEGUN, I.A., Handbook of Mathematical Functions, Dover Publications, New York, 1970.

2. ANDREWS, G.E., The Theory of Applications, Encyclopedia of Math. and its Applications, Vol. 2, Addison Wesly, 1976.

3. APOSTOL, T.M. \& ZUCKERMAN, H.S., On the functional equation $F(m n) F(m, n)$ $=F(m) F(n) f((m, n))$, Pac. J. Math. 14 (1964), 371-384.

4. CAMPBELL, G.B., A new class of infinite products and Euler's totient, Int. J. of Math. and Math. Sci. (To appear).

5. CHANDRASEKHARAN, K., Arithmetical Functions, Vol. 67, Springer-Verlag, New York, 1970.

6. EDWARDS, H.M., Riemann's zeta function, Pure and Applied Mathematics, Vol. 58, New York Academic Press, 1974.

7. ELLIOTT, P.D.T.A., Arithmetic functions and integer products, Grundlehren der Mathematischen Wissenschaften, Vol. 272, Springer-Verlag, New York, 1985.

8. HARDY, G.H., Note on Ramanujan's arithmetical function $\tau(n)$, Collected Papers of G.H. Hardy, Vol. 2, Oxford at the Clarendon Press (1967), 358.

9. HARDY, G.H., Note on Ramanujan's trigonometrical function $c_{g}(n)$ and certain series of mathematical functions, Collected Papers of G.H. Hardy, Vol. 2, Oxford at the Clarendon Press (1967), 320.

10. HARDY, G.H., Ramanujan, Chelsea Publishing Company, New York (1940), 139-143.

11. HARDY, G.H. \& WRIGHT, E.M., An Introduction to the Theory of Numbers, Oxford: Clarendon Press, New York, Oxford University Press, 1979.

12. LOSSERS, O.P., The generalized totient function, solution to problem No. 6446, Amer. Math. Monthly (1985), 434.

13. RAMANUJAN, S., On certain trigonometrical sums and their application to the theory of numbers, Collected Papers of S. Ramanujan, Cambridge University Press, Cambridge (1927), 179-199; reprinted by Chelsea, New York, 1962.

14. SIVARAMAKRISHNAN, R., Classical theory of arithmetic functions, Monographs and Textbooks in Pure and Applied Mathematics, Vol. 126, Marcel Dekker Inc., New York, 1962.

15. TITCHMARSH, E.C., The Theory of the Riemann Zeta Function, Oxford at the Clarendon Press, 1951. 


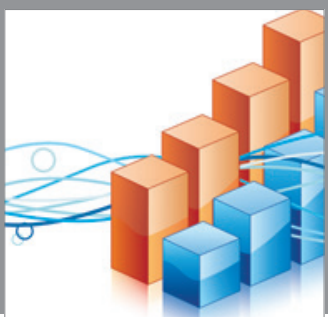

Advances in

Operations Research

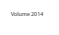

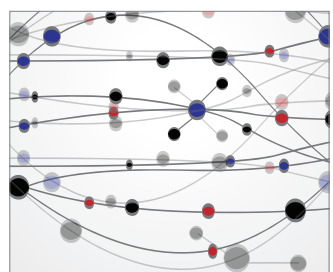

\section{The Scientific} World Journal
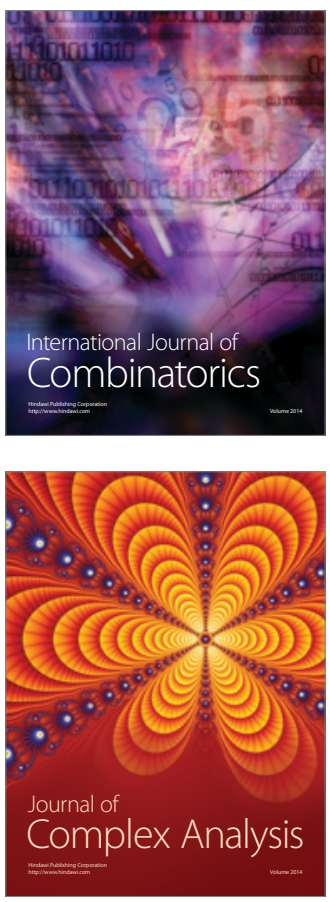

International Journal of

Mathematics and

Mathematical

Sciences
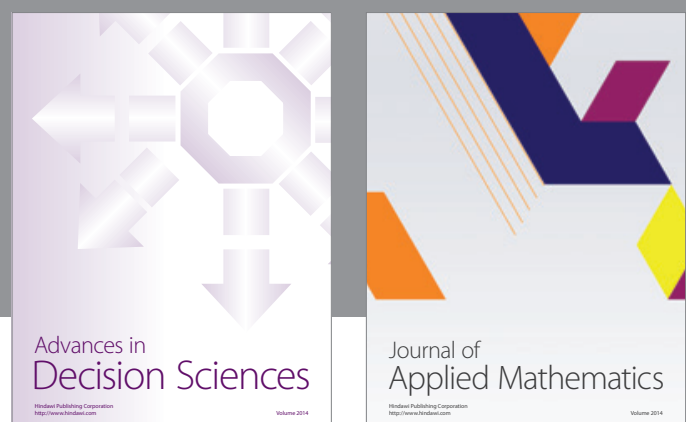

Journal of

Applied Mathematics
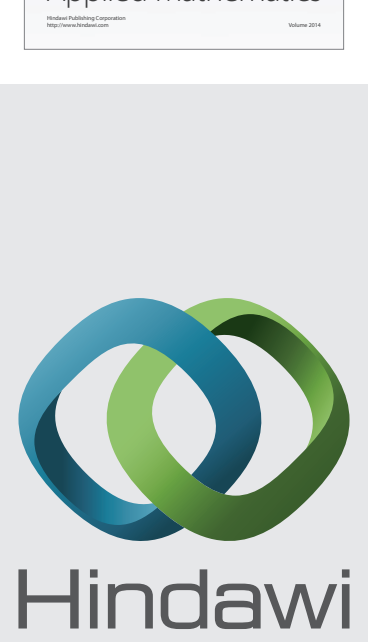

Submit your manuscripts at http://www.hindawi.com
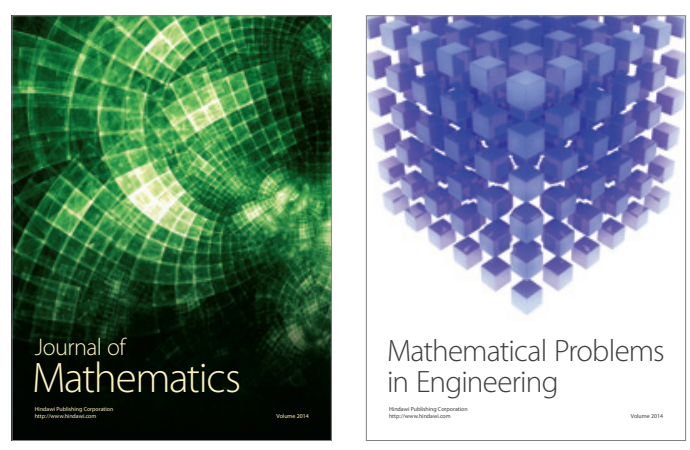

Mathematical Problems in Engineering
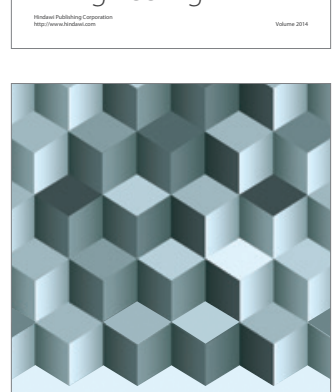

Journal of

Function Spaces
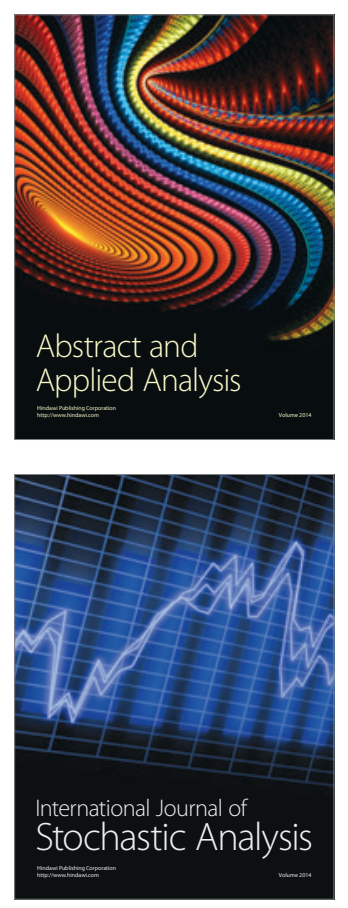

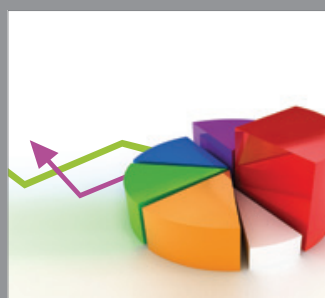

ournal of

Probability and Statistics

Promensencen
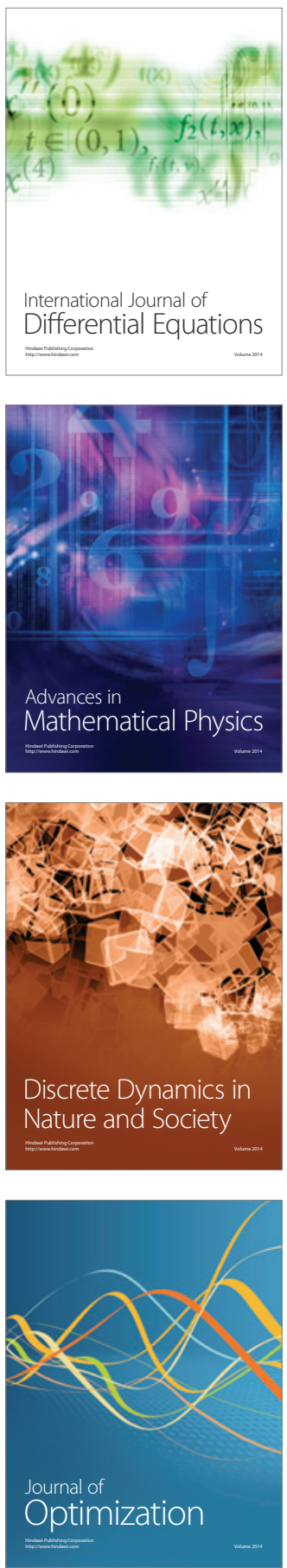